\title{
Associative Memory Stored by Functional Novel Pathway rather than Modifications of Preexisting Neuronal Pathways
}

\author{
Volko A. Straub, Ildiko Kemenes, Michael O'Shea, and Paul R. Benjamin \\ Sussex Centre for Neuroscience, School of Biology and Environmental Science, University of Sussex, Falmer, Brighton BN1 9QG, United Kingdom
}

Associative conditioning involves changes in the processing pathways activated by sensory information to link the conditioned stimulus (CS) to the conditioned behavior. Thus, conditioning can recruit neuronal elements to form new pathways for the processing of the CS and/or can change the strength of existing pathways. Using a behavioral and systems level electrophysiological approach on a tractable invertebrate circuit generating feeding in the mollusk Lymnaea stagnalis, we identified three independent pathways for the processing of the CS amyl acetate used in appetitive conditioning. Two of these pathways, one suppressing and the other stimulating feeding, mediate responses to the CS in naive animals. The effects of these two pathways on feeding behavior are unaltered by conditioning. In contrast, the CS response of a third stimulatory pathway is significantly enhanced after conditioning, becoming an important contributor to the overall CS response. This is unusual because, in most of the previous examples in which naive animals already respond to the CS, memory formation results from changes in the strength of pathways that mediate the existing response. Here, we show that, in the molluscan feeding system, both modified and unmodified pathways are activated in parallel by the CS after conditioning, and it is their integration that results in the conditioned response.

Key words: learning; memory; conditioning; Lymnaea; chemosensory processing; sensory integration

\section{Introduction}

Existing models of associative memory formation can be divided into two categories. In the first, before conditioning, the conditioned stimulus (CS) is neutral with respect to the behavior to be conditioned. Here associative conditioning requires the recruitment of new processing pathways to link the CS to the conditioned behavioral response [e.g., rabbit eyeblink conditioning (Thompson, 2005)]. In the second category, the CS is not neutral but evokes a response before conditioning that is related to the conditioned behavior. In this case, associative conditioning can derive from a strengthening or weakening of existing neural pathways [e.g., conditioning of Aplysia gill withdrawal reflex (Antonov et al., 2001)]. The existence of these two categories suggests that functionally novel processing pathways are only recruited when, before conditioning, the CS does not evoke a response related to the conditioned behavior. In contrast, in systems in which there is an existing response to the CS that is altered in its amplitude by conditioning, it appears more likely that this is attributable to changes in existing neural pathways.

Chemical appetitive conditioning in the pond snail Lymnaea stagnalis is an established model system for the study of the cellular mechanisms that underlie associative memory formation (Kemenes et al., 2002; Ribeiro et al., 2003; Fulton et al., 2005). In

\footnotetext{
Received Sept. 19, 2005; revised March 6, 2006; accepted March 11, 2006.

This work was supported by the Biotechnology and Biological Sciences Research Council (United Kingdom).

Correspondence should be addressed to Volko A. Straub at his present address: Department of Cell Physiology and Pharmacology, University of Leicester, Maurice Shock Medical Sciences Building, P.O. Box 138, University Road, Leicester LE1 9HN, UK. E-mail: vs64@le.ac.uk.

DOI:10.1523/JNEUROSCI.0489-06.2006

Copyright $\odot 2006$ Society for Neuroscience $\quad$ 0270-6474/06/264139-08\$15.00/0
}

conditioning of feeding behavior, amyl acetate (the CS) is paired with sucrose, [the unconditioned stimulus (US)], resulting in the formation of an associative long-term memory (Alexander et al., 1984). Although the CS has been reported to be neutral with respect to feeding (Alexander et al., 1982; Audesirk et al., 1982), we show here that, before conditioning, amyl acetate can both excite and inhibit feeding behavior depending on its concentration. Our knowledge of the Lymnaea feeding system (Benjamin and Elliott, 1989; Benjamin et al., 2000; Kemenes et al., 2001; Straub et al., 2002) allowed us to identify the neural pathways serving the excitatory and inhibitory effects on feeding in naive animals. Their effects on feeding, however, are unaltered by associative conditioning. Contrary to expectations, our experiments also show that a new feeding stimulatory pathway that was previously too weak for detection is significantly enhanced by conditioning, leading to its functional recruitment to the CS response. We show that the behavioral expression of the associative memory derives from the parallel activation of the enhanced CS processing pathway and the existing inhibitory and excitatory CS pathways. The integration of all three pathways accounts for the conditioned feeding response.

\section{Materials and Methods}

Animals. All experiments described here were performed using adult (4-5 month old) pond snails, L. stagnalis. The animals were bred and raised in the Lymnaea breeding facilities at the University of Sussex, where they were kept in copper-free ( $\mathrm{Cu}$-free) water at a temperature of $20^{\circ} \mathrm{C}$ in a $12 \mathrm{~h} \mathrm{light/dark} \mathrm{cycle} \mathrm{and} \mathrm{were} \mathrm{fed} \mathrm{a} \mathrm{diet} \mathrm{of} \mathrm{lettuce} \mathrm{and} \mathrm{fish} \mathrm{food.}$

Testing of CS effects on feeding behavior in naive animals. Snails were starved for $4 \mathrm{~d}$ before testing. For testing, individual naive animals were placed in $15 \mathrm{~cm}$ Petri dishes filled with $90 \mathrm{ml}$ of $\mathrm{Cu}$-free water. After 10 min, a $5 \mathrm{ml}$ neutral water stimulus was added to all dishes, and the 
$\mathrm{Ai}$

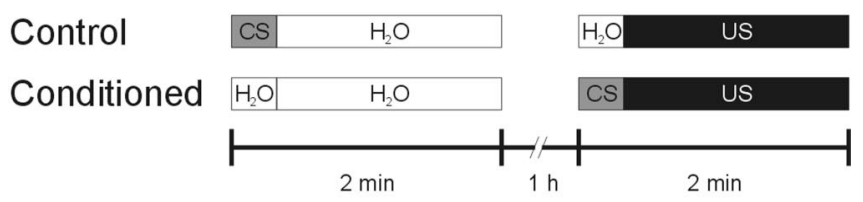

$24 \mathrm{~h}$

Aii

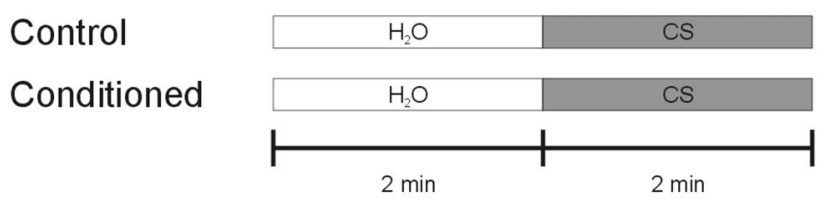

B

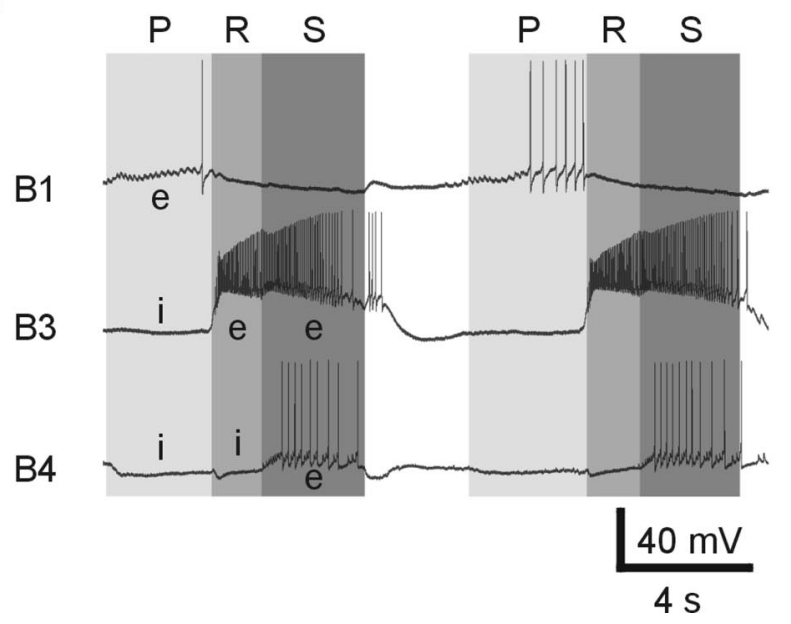

Figure 1. Chemical appetitive conditioning procedure and fictive feeding activity. Ai, Aii, Schematic representation of the training procedures used in the chemical appetitive conditioning of Lymnaea feeding behavior. For more details, see description of the conditioning procedure in Materials and Methods. $\boldsymbol{B}$, Example record of two cycles of fictive feeding during US applications to the lips. Fictive feeding activity was monitored by recording buccal neurons B1, $B 3$, and B4. The three phases of a fictive feeding cycle are indicated by shaded areas and are labeled P (protraction), R (rasp), and S (swallow). Characteristic excitatory (e) and inhibitory (i) synaptic inputs to feeding neurons during a feeding cycle are indicated on the record.

number of mouth openings followed by the protraction of the radula that constitute one feeding movement, or "rasp," was counted for a period of $2 \mathrm{~min}$. This provided a baseline of feeding activity during a $2 \mathrm{~min}$ period after disturbance of the dish solution. Afterward, the CS ( $5 \mathrm{ml}$ of amyl acetate; concentration, $0.04,0.08$, and $0.4 \%$ ) was added to the dish (final amyl acetate concentrations in the dish, 0.002, 0.004, and 0.02\%), and rasps were counted for another 2 min period.

One-trial appetitive conditioning of Lymnaea. One-trial appetitive conditioning of Lymnaea was performed using a training procedure based on previously described protocols (Kemenes et al., 2002). Two groups of animals were included in each experiment that were starved for $4 \mathrm{~d}$ before training. During training, the conditioned group received a paired presentation of the CS amyl acetate and the US sucrose, whereas the control group received a single presentation of the CS amyl acetate followed by a single presentation of the US sucrose $1 \mathrm{~h}$ later. The behavioral training and testing procedure is summarized in Figure 1, Ai and Aii.

For training, individual snails were positioned in the center of a $15 \mathrm{~cm}$ Petri dish filled with clean $\mathrm{Cu}$-free water $(90 \mathrm{ml})$ and left for $10 \mathrm{~min}$. At this point, the CS ( $5 \mathrm{ml}$ of $0.08 \%$ amyl acetate; final concentration in the dish, $0.004 \%$ ) was added to dishes containing animals in the control group, and a neutral water stimulus $(5 \mathrm{ml})$ was added to dishes containing animals in the conditioned group. After $20 \mathrm{~s}$, a second neutral water stimulus ( $5 \mathrm{ml}$ ) was added to all dishes, so that the dishes contained 100 $\mathrm{ml}$ of solution. The animals were removed from these dishes $2 \mathrm{~min}$ after the addition of the first stimulus and returned to a tank containing clean water.

After 50 min, individual animals were again put in clean Petri dishes filled with water $(90 \mathrm{ml})$ and left for $10 \mathrm{~min}$. At this point, a neutral water stimulus $(5 \mathrm{ml})$ was added to dishes containing animals in the control group, and the CS ( $5 \mathrm{ml}$ of $0.08 \%$ amyl acetate) was added to dishes with animals from the conditioned group. After $20 \mathrm{~s}$, the US ( $5 \mathrm{ml}$ of $0.4 \mathrm{M}$ sucrose; final concentration in dish, $0.02 \mathrm{M}$ ) was added to all dishes. Again, the animals were removed from the dishes $2 \mathrm{~min}$ after the addition of the first stimulus, that is, the neutral water stimulus or CS, and transferred to a tank containing clean water to rinse off any chemical stimuli. After $10 \mathrm{~min}$, the animals were transferred to another tank containing clean water, in which they remained until testing. This training protocol ensured that both groups received the same amount of handling and the same amount of exposure to both the CS and US. We also tested whether CS application alters the effect of the US on feeding behavior during the training phase by counting the number of rasps during US application in a control and conditioned group. The feeding stimulatory effect of the US was unaltered by the presence of the CS in the conditioned group compared with the control group that received the US alone (control group, $30 \pm 2.4 \mathrm{rasps} / 2 \mathrm{~min}, n=18$; conditioned group, $31.4 \pm 1.5 \mathrm{rasps} / 2 \mathrm{~min}$, $n=19 ; t$ test, $p=0.62$ ). Thus, the only difference between the two groups is that the conditioned group received a paired presentation of the US and CS, whereas the control group received an explicitly unpaired presentation of the two stimuli.

The effect of the appetitive conditioning was tested "blind" $24 \mathrm{~h}$ after training (Fig. 1 Aii). For testing, individual animals were placed for 10 $\mathrm{min}$ in $15 \mathrm{~cm}$ Petri dishes filled with $\mathrm{Cu}$-free water $(90 \mathrm{ml})$. Each animal was then presented with a neutral water stimulus ( $5 \mathrm{ml}$ of water added to the dish), and the number of rasps was counted for a period of $2 \mathrm{~min}$. After the 2 min observation period, the CS ( $5 \mathrm{ml}$ of amyl acetate; concentration, $0.04,0.08$, and $0.4 \%$ ) was added to the dish (final amyl acetate concentrations in the dish, $0.002,0.004$, and $0.02 \%$ ), and the number of rasps was counted for another 2 min period. Afterward, animals were rinsed and returned to clean tanks.

Monitoring effects of chemosensory stimulation on fictive feeding activity in semi-intact preparations. Semi-intact preparations were prepared from naive, control, and conditioned animals as indicated in Results. CS and US responses in these preparations were recorded 1-4 d after behavioral training, and all experiments were performed blind (i.e., the experimenter was not aware of the training history of the preparation). All dissections and recordings were performed in standard Lymnaea saline (Straub et al., 2002).

Two types of semi-intact preparations, whole and reduced CNS, were used for the experiments described in this paper. The whole CNS preparation consisted of the head of the animal containing the mouth region, lips, and tentacles, the entire circumesophageal ganglionic ring, the buccal ganglia, and a long stretch of esophagus (see Fig. $2 \mathrm{Bi}$ ). In this preparation, the median and superior lip nerves and the tentacle nerves are intact, forming the connection from the head structure to the cerebral ganglia, whereas the cerebral-buccal connectives link the cerebral ganglia to the buccal ganglia. The stretch of esophagus is connected directly to the buccal ganglia via the dorsal buccal nerves. The reduced CNS preparation was identical to the whole CNS preparation, with the only difference that the cerebral-pedal and cerebral-pleural connectives were cut to sever all connections between the cerebral ganglia and the remaining circumesophageal ganglia (see Fig. 3Ai).

In both types of preparations, the distal end of the esophagus was stretched over a short length of plastic tubing to enable selective superfusion of the internal esophageal surface. The plastic tubing was connected to a manifold, enabling the rapid switching between solutions using a computer-controlled, custom-built superfusion system. A second superfusion pipette was placed directly in front of the mouth region 
of the preparation and was connected to separate channels of the superfusion system, enabling the selective application of stimulus solutions to either the esophagus or lips/tentacles. Both sites were constantly superfused with standard Lymnaea saline at a rate of $1.5-2 \mathrm{ml} / \mathrm{min}$ throughout the experiments. For stimulus application, the superfusion was rapidly switched to either of the following stimulus solutions: $0.02 \mathrm{M}$ sucrose (US) or $0.002,0.004$, and $0.02 \%$ amyl acetate (all made up in standard Lymnaea saline). Control experiments confirmed that selective superfusion of either of the two sites evoked no significant response on the other site. Furthermore, cutting of sensory nerves between the lips and cerebral ganglia and the esophagus and buccal ganglia abolished all effects of US or CS application on fictive feeding activity, indicating that US and CS effects on neuronal activity are mediated via stimulation of the chemosensory structures.

Neuronal activity in these preparations was recorded using sharp intracellular microelectrodes filled with KAc (3 M; electrode resistance, 30-40 M $\Omega$ ). Electrical signals were amplified using Neurolog NL102 (Digitimer, Hertfordshire, UK) and AxoClamp 2B amplifiers (Molecular Devices, Palo Alto, CA), digitized using a CED 1401 interface (Cambridge Electronics Design, Cambridge, UK), and displayed and stored on a personal computer running Spike2 software (Cambridge Electronics Design).

Fictive feeding activity in these preparations was monitored by recording neuronal activity in the buccal feeding motoneurons B1, B3, and B4. These three types of buccal neurons are visually identifiable in the intact ganglia and receive distinct patterns of synaptic inputs during fictive feeding activity (Fig. $1 B$ ). B1 neurons receive an excitatory synaptic input that causes a depolarization of the membrane potential during the protraction phase. In contrast, B3 and B4 neurons receive inhibitory synaptic inputs during the protraction phase (Benjamin and Elliott, 1989). The rasp phase is characterized by a strong excitation of B3 neurons and a simultaneous strong inhibition of B4 neurons. Finally, the swallow phase is indicated by a second phase of depolarization of the B3 neuron and the depolarization of the $\mathrm{B} 4$ neurons that triggers a burst of action potentials after the strong rasp phase inhibition. Thus, simultaneously monitoring activity in these motoneuron types enables the unambiguous identification of fictive feeding cycles in semi-intact preparations. The number of feeding cycles that fulfilled these criteria was counted for a $2 \mathrm{~min}$ period immediately before stimulus application and compared with feeding activity during a 2 min period of stimulus application.

Previous experiments had shown that simultaneous US application to the lips and the esophagus is a very reliable feeding stimulus in semiintact preparations. Therefore, we only included preparations in the analysis that showed an increase in fictive feeding activity in response to this form of stimulation to eliminate preparations in which sensory pathways were damaged during the dissection procedure. Based on this criterion, $14 \%$ of preparations ( 27 of 199 ) were excluded from the analysis. All analysis of the recordings from semi-intact preparations was performed blind.

Standard statistical analyses ( $t$ test, ANOVA, etc.) as indicated in Results were performed using the SPSS software package (SPSS, Chicago, IL).

\section{Results}

\section{Identification of CS pathways in naive snails}

Amyl acetate has been described previously as a neutral feeding stimulus (Alexander et al., 1982; Audesirk et al., 1982). However, by applying different concentrations of the CS, we identified excitatory and inhibitory feeding responses in naive snails. Snails usually perform spontaneous feeding movements so it is possible to measure both increases and decreases in feeding rates from this "baseline" value. At the concentration of amyl acetate used previously for conditioning $(0.004 \%)$, there is no effect on feeding ("neutral stimulus") (Fig. 2A). However, applying the CS at half that concentration $(0.002 \%$ amyl acetate $)$ significantly stimulates feeding (paired $t$ test, $p<0.05 ; n=24$ ), whereas increasing the concentration fivefold to $0.02 \%$ amyl acetate inhibits spontaneous feeding (paired $t$ test, $p<0.05 ; n=24$ ).
These behavioral experiments suggested that there are both excitatory and inhibitory chemosensory pathways in the Lymnaea feeding system that are activated at different CS concentrations. We identified these pathways by making semi-intact preparations that allow application of the CS to different sensory surfaces and simultaneous electrophysiological recording of the feeding motor program in the buccal ganglia. In Lymnaea, chemosensory information from external (lips) and internal (esophagus) sensory structures is processed by anatomically distinct pathways (Fig. 2 Bi) (see also Fig. 5A). External chemosensory information is integrated in the cerebral ganglia before it is transferred to the feeding central pattern generator in the buccal ganglia (Straub et al., 2004). In contrast, internal chemosensory information enters the buccal ganglia directly, in which it affects the feeding pattern generator. The existence of this direct chemosensory pathway was confirmed in preliminary experiments, which demonstrated that US application to the esophagus induces feeding activity in minimal preparations consisting only of the esophagus and buccal ganglia (Straub et al., 2003).

In the semi-intact preparation, we were able to superfuse selectively chemosensory epithelia located on the lips and the internal surface of the esophagus (Fig. $2 \mathrm{Bi}$ ). The effect of stimulating these structures was measured as changes in fictive feeding activity in response to the stimulus. Fictive feeding activity was monitored by recording neuronal activity in feeding motoneurons that have been shown to receive characteristic synaptic inputs during feeding cycles in the intact animal (Rose and Benjamin, 1979; Benjamin and Elliott, 1989).

The fictive feeding rate during CS application to the lips was significantly reduced at the highest concentration $(0.02 \%$; paired $t$ test, $p<0.05 ; n=12$ ) (Fig. $2 \mathrm{Bii}, \mathrm{Ci}$ ). Additional tests with lower CS concentrations ( 0.004 and $0.002 \%)$ applied to the lips had no significant effect on fictive feeding activity (paired $t$ tests, $p>$ $0.05 ; n=12$ ) (Fig. 2 Bii). In contrast, CS application to the esophagus stimulated fictive feeding activity even at the lowest concentration tested (0.002\%; paired $t$ tests, $p<0.05 ; n=7$ ) (Fig. 2 Biii,Cii). Similar feeding stimulatory effects were observed in response to the application of higher CS concentrations $(0.004$ and $0.02 \%$ ) to the esophagus (paired $t$ tests, $p<0.01 ; n=7$ ) (Fig. 2 Biii). These results confirm the existence of separate CS processing pathways with distinct activation thresholds in naive snails that have inhibitory (lips) and stimulatory (esophagus) effects on fictive feeding responses. Given that spontaneous feeding ensures that both sensory structures are stimulated by the CS in the intact snail, it is reasonable to conclude that the esophagus will mediate the increase in behavioral feeding at the lowest CS concentration and lip stimulation the inhibition of feeding seen at the highest concentration (Fig. $2 \mathrm{~A}$ ).

It was important to characterize the CNS pathway responsible for the inhibitory component of the lip CS response in more detail to assess its role in learning. During the tests for the effects of high CS concentrations on feeding behavior, it was noticed that the inhibition of feeding activity was accompanied by aversive responses such as withdrawal. Defensive and feeding behaviors are mutually exclusive in Lymnaea (Kemenes et al., 1986; Kyriakides and McCrohan, 1988; Winlow et al., 1992), suggesting that the suppression of feeding activity by high CS concentrations is the indirect result of the activation of withdrawal circuits in the CNS. Because neurons of the withdrawal response are located in the nonfeeding ganglia of the CNS (Ferguson and Benjamin, 1991; Inoue et al., 1996), we supposed that the feeding suppressive effects were the result of negative feedback that originate from the withdrawal circuitry located outside the cerebral and 


\section{A Naïve Animals - Behavior}

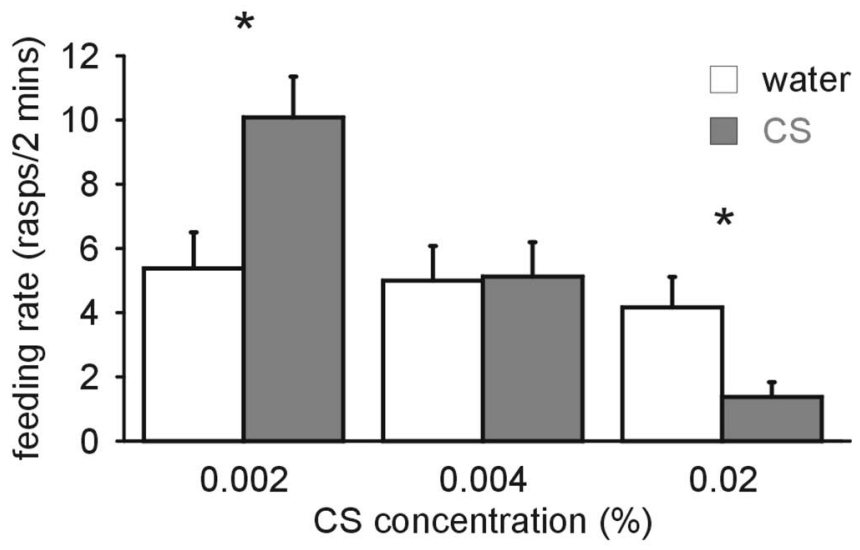

\section{Bi Naïve whole CNS preparation}

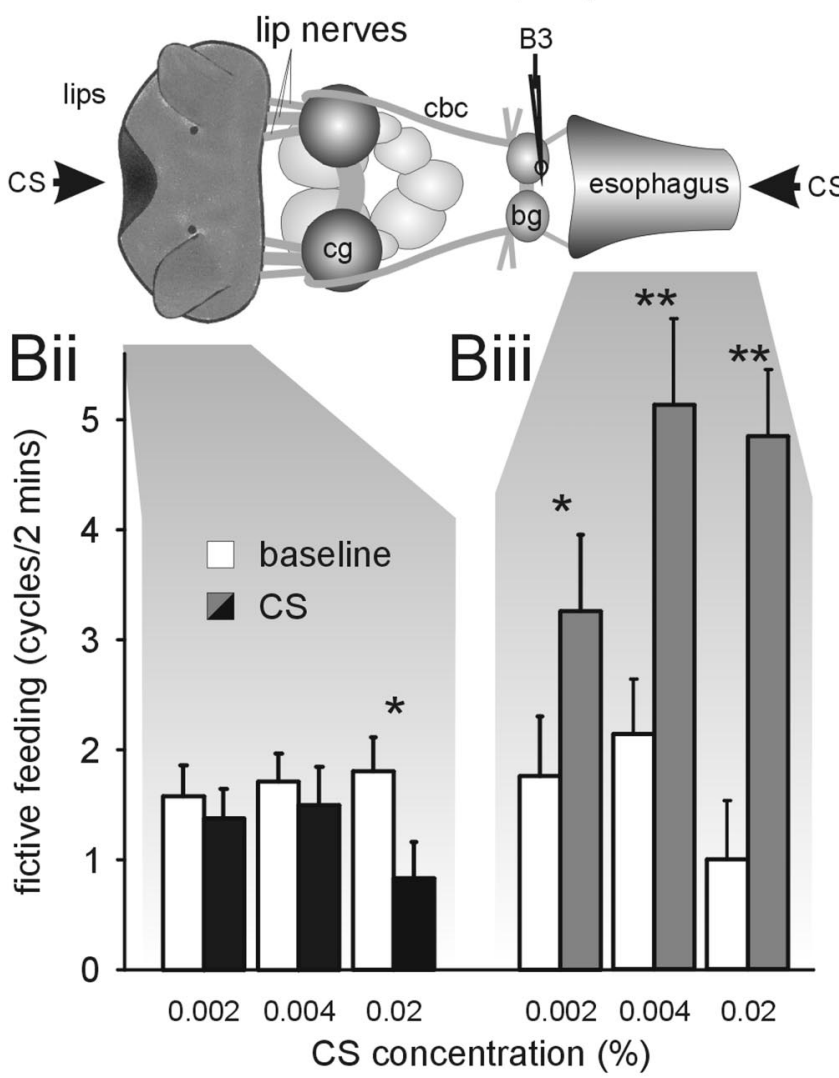

\section{Ci Suppression of fictive feeding}

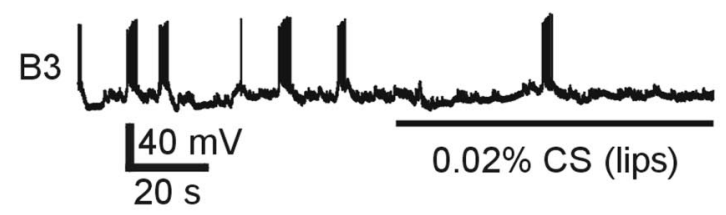

\section{Cii Stimulation of fictive feeding}

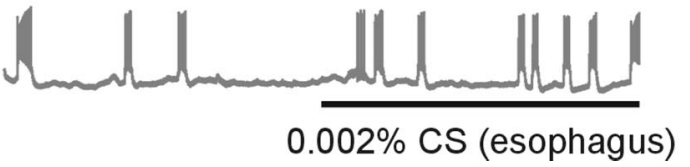

buccal ganglia. To test this hypothesis, we removed all of the CNS apart from the cerebral and buccal ganglia from the semi-intact preparation (Fig. 3Ai, reduced CNS preparation). This left the lip-cerebral-buccal ganglia pathway and the more direct esophageal-buccal ganglia pathway intact (see Fig. $5 \mathrm{~A}$ ) and available for CS stimulation.

Unlike the semi-intact preparation with the intact CNS (whole CNS preparation), CS application to the lips in the reduced CNS preparation produced no significant inhibition of fictive feeding activity (paired $t$ test, $p>0.05 ; n=34$ ) (Fig. 3Aii). This neutral response in the reduced CNS preparation was significantly different from the feeding suppressive effect of the same stimulus observed in the whole CNS preparation $(t$ test, $p<0.05)$ (Fig. $2 \mathrm{Bii}$ ). Additional tests using the $\mathrm{CS}$ at lower concentrations $(0.002$ and $0.004 \%)$ also produced no significant changes in fictive feeding rates $[\Delta$ fictive feeding, $0.002 \%, 0.6 \pm 0.7$ cycles $/ 2$ $\min (n=10)$, paired $t$ test, $p>0.05 ; 0.004 \%,-0.1 \pm 0.4$ cycles $/ 2$ $\min (n=10)$, paired $t$ test, $p>0.05]$. Thus, this experiment provides direct evidence that ganglion excision interrupts the CS processing pathway from the lips mediating the suppression of feeding behavior.

In contrast, ganglion excision had no significant effect on the feeding stimulatory effect of CS application to the esophagus (Fig. 3Aiii) when compared with the responses in the whole CNS preparation ( $t$ test, $p>0.05$ ) (Fig. 2Cii). Neither did ganglion removal affect the ability of US application to the lips to produce a feeding stimulatory effect in reduced CNS preparations (increase in fictive feeding by $2.2 \pm 0.6$ cycles $/ 2 \mathrm{~min} ; n=13$ ), which is similar to the US response in the whole CNS preparation (increase in fictive feeding by $1.3 \pm 0.4$ cycles $/ 2 \mathrm{~min} ; n=36 ; t$ test, $p>0.05)$. These experiments clearly show that the inhibitory response to stimulation of the lips by the CS requires parts of the CNS located outside of the feeding ganglia (rest of CNS in Fig. 5). The stimulatory effects of the US on the lips are not affected by ganglion removal, showing that the normal feeding-activation pathways are retained in the reduced CNS preparation.

\footnotetext{
Figure 2. Identification of two separate $C S$ processing pathways in naive animals. $\boldsymbol{A}$, Concentration dependence of $\mathrm{CS}$ effects on feeding behavior in naive animals indicates the existence of two independent CS processing pathways with different activation thresholds. At $0.004 \%$, the CS is a behaviorally "neutral" stimulus that does not significantly alter the spontaneous feeding activity. However, $0.002 \%$ CS results in a significant increase in feeding activity, and $0.02 \% \mathrm{CS}$ significantly inhibits feeding activity. Statistical analysis revealed a strong negative correlation between the $\mathrm{CS}$ concentration and its effect on feeding (Spearman's rank correlation: $\rho=-0.59 ; p<0.01) .{ }^{*} p<0.05 . B, C$, Opposing effects of $C S$ application to the lips and esophagus on fictive feeding activity in semi-intact preparations. $\boldsymbol{B i}$, Schematic diagram of whole CNS semi-intact preparation. The semi-intact preparation consisted of the lip structures, the esophagus, and the whole CNS, including the cerebral ganglia (cg) and buccal ganglia (bg). A custom-built superfusion system enabled the separate stimulation of the lips and the inside of the esophagus with the $C S$. The $C S$ effects on fictive feeding activity were monitored by recording electrical activity in buccal feeding motoneurons including the B3 using standard intracellular recording techniques. cbc, Cerebral buccal connectives. Bii, The $0.02 \%$ CS application to the lips for 2 min significantly suppressed fictive feeding activity (CS) compared with the $2 \mathrm{~min}$ period before stimulus application (baseline). Additional tests with lower CS concentrations $(0.004$ and $0.002 \%$ ) had no significant effect on fictive feeding activity (paired $t$ tests, $p>0.05$; $n=12)$. Biii, CS application to the esophagus significantly increased fictive feeding over baseline activity at all concentrations tested. ${ }^{*} p<0.05$; ${ }^{*} p<0.01$. Ci, Sample record from B3 motoneuron showing suppression of fictive feeding as indicated by reduction of $B 3$ activity from four bursts before $0.02 \% \mathrm{CS}$ application to the lips to a single burst during $\mathrm{CS}$ application. Cii, Sample record from $B 3$ motoneuron showing stimulation of fictive feeding as indicated by the increase in B3 activity from three bursts before $0.002 \% \mathrm{CS}$ application to the esophagus to seven bursts during CS application.
} 
Ai Naïve reduced CNS preparation

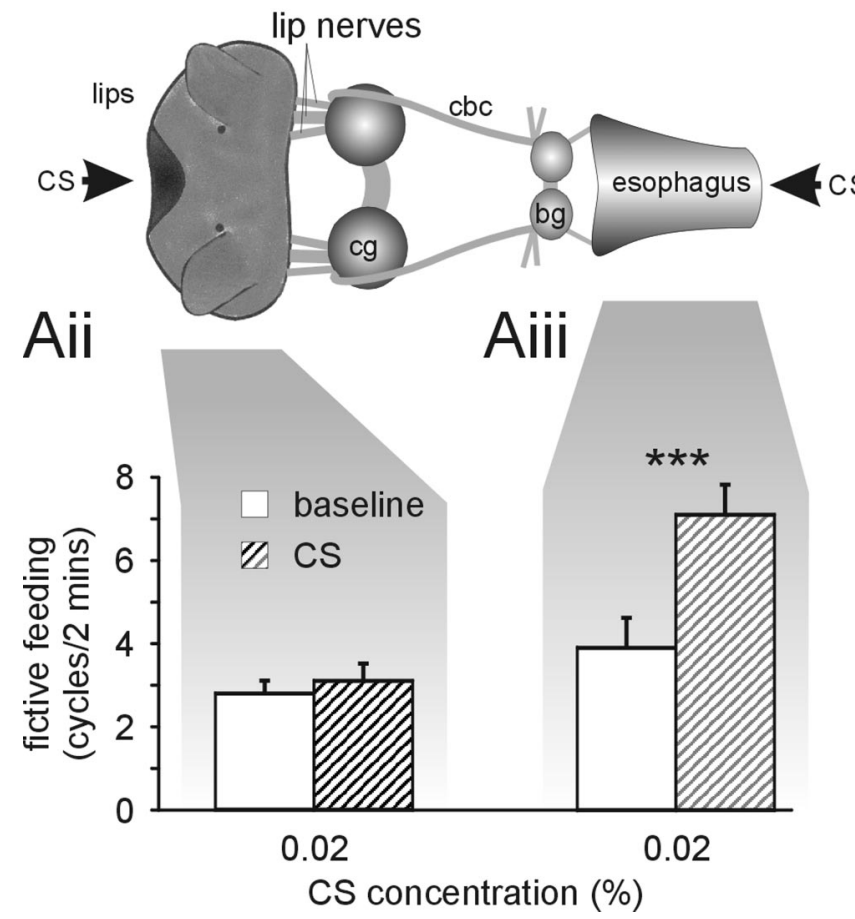

Figure 3. Inhibitory effect of CS application to the lips requires whole CNS. Ai, Schematic diagram of reduced CNS semi-intact preparation. The reduced CNS preparation is similar to the semi-intact preparation shown in Figure 1 but lacks all central ganglia apart from the cerebral ganglia (cg) and the buccal ganglia (bg). This left direct lip-cerebral- buccal and esophagealbuccal pathways intact but removed all indirect pathways requiring neuronal elements outside the cerebral- buccal ganglia. cbc, Cerebral buccal connectives. Aii, CS application (0.02\%) to the lips of reduced semi-intact preparations does not significantly alter fictive feeding activity during the 2 min application (CS) compared with the 2 min before stimulus application (baseline). Aiii, CS application (0.02\%) to the esophagus of reduced CNS preparations causes a highly significant increase in baseline fictive feeding activity by $3.2 \pm 0.4$ cycles $/ 2 \mathrm{~min}$ (paired $t$ test, $p<0.001 ; n=10$ ), which is similar to the feeding stimulatory effect of $0.02 \%$ CS application in the whole CNS preparation ( $3.9 \pm 0.7$ cycles $/ 2$ min; $n=7 ; t$ test, $p>0.05$ ). ${ }^{* * *} p<0.001$.

\section{Appetitive conditioning results in the strengthening of a} feeding stimulatory pathway from the lips

Behavioral conditioning produces an increase in the feeding response to the CS. At the highest CS concentration $(0.02 \%)$, conditioned animals responded with a significant increase in feeding rate (paired $t$ test, $p<0.05 ; n=39$ ), reversing the feeding inhibitory effect observed at this CS concentration in naive and control animals (Fig. 4A). Feeding rates during CS application in conditioned animals were also significantly higher than in unpaired control animals at the other concentrations tested $(0.002 \%$, control, $7.9 \pm 1.1 \mathrm{rasps} / 2 \mathrm{~min}, n=38$; conditioned, 12.3. $\pm 1.4, n=$ 39; $0.004 \%$, control, $5.9 \pm 0.9 \mathrm{rasps} / 2 \mathrm{~min}, n=39$; conditioned, 10.9. $\pm 1.3, n=37$; $t$ tests, $p<0.05$ ). This conditioned response could be the result of alterations in either of the two CS processing pathways that have been shown to significantly affect feeding behavior in naive animals. Experiments investigating lip responses in reduced semi-intact preparations, however, demonstrated the strengthening of a lip stimulatory pathway that was activated by the CS and contributed to the conditioned response. The previous experiment (Fig. 3) on naive snails showed that reducing the preparation removes the lip inhibitory response to the CS but left the direct pathway from the lips to the cerebral ganglia intact and available for testing after learning. After behavioral conditioning, reduced preparations showed a significant

\section{A Conditioned animals Behavior}

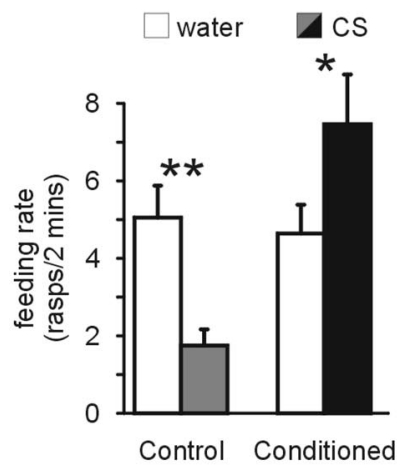
Conditioned
reduced CNS preparation
CS application to lips
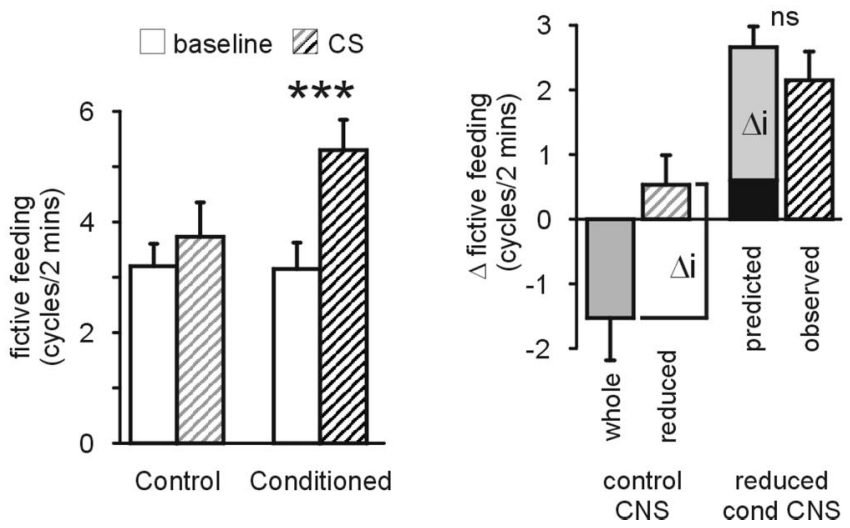

Figure 4. Conditioning induces a novel feeding stimulatory lip CS pathway. A, Conditioning reverses feeding suppressive $C S$ effect in the intact animal. Animals that received an explicitly unpaired presentation of the CS and US during conditioning (Control) showed a significant suppression of feeding activity during the $0.02 \%$ CS application (CS) compared with the water score (water). This feeding suppressive effect was reversed in conditioned animals, in which $0.02 \%$ CS caused a significant increase in feeding activity (Conditioned). $\boldsymbol{B}, \mathrm{CS}$ application $(0.02 \%)$ to the lips causes a significant increase in fictive feeding activity in reduced CNS preparations (see Fig. 2Ai) from conditioned animals but not from control animals. C, Conditioning reverses feeding suppressive effect of CS in whole CNS preparations. CS application $(0.02 \%)$ to the lips causes a significant reduction in fictive feeding activity in whole CNS preparations from control animals but not from conditioned animals. Baseline, Fictive feeding activity during 2 min before CS application; CS, fictive feeding activity during 2 min of CS application. D, Conditioning does not change the inhibitory pathway that mediates feeding suppressive $C S$ effect in naive animals. The feeding suppressive effect of $\mathrm{CS}$ application to the lips is only present in the whole CNS (whole control CNS) but not in the reduced CNS preparation (reduced control CNS). Therefore, the absolute difference between the CS effect in whole CNS and reduced CNS preparations from control animals provides an estimate of the inhibitory component $(\Delta i)$. If this inhibitory component is unchanged by conditioning, adding $\Delta i$ to the observed CS effect in whole CNS preparations from conditioned animals (predicted) should provide a good prediction for the CS effect in reduced CNS preparations from conditioned animals (reduced cond CNS) that lack the inhibitory pathway (observed). This is in fact the case, because there is no significant difference between the predicted and observed CS effect in reduced CNS preparations from conditioned animals ( $t$ test, $p<0.05$ ). This strongly suggests that conditioning does not alter the inhibitory CS pathway. ${ }^{*} p<0.05$; ${ }^{* *} p<0.01$; ${ }^{* * *} p<0.001$. ns, Not significant.

increase in fictive feeding activity in response to $0.02 \%$ CS application to the lips (paired $t$ test, $p<0.001 ; n=20$ ) (Fig. $4 B$ ). As expected, reduced CNS preparations made from unpaired control animals showed no significant change in the level of baseline fictive feeding activity (paired $t$ test, $p=0.26$ ) (Fig. 4B). Further- 


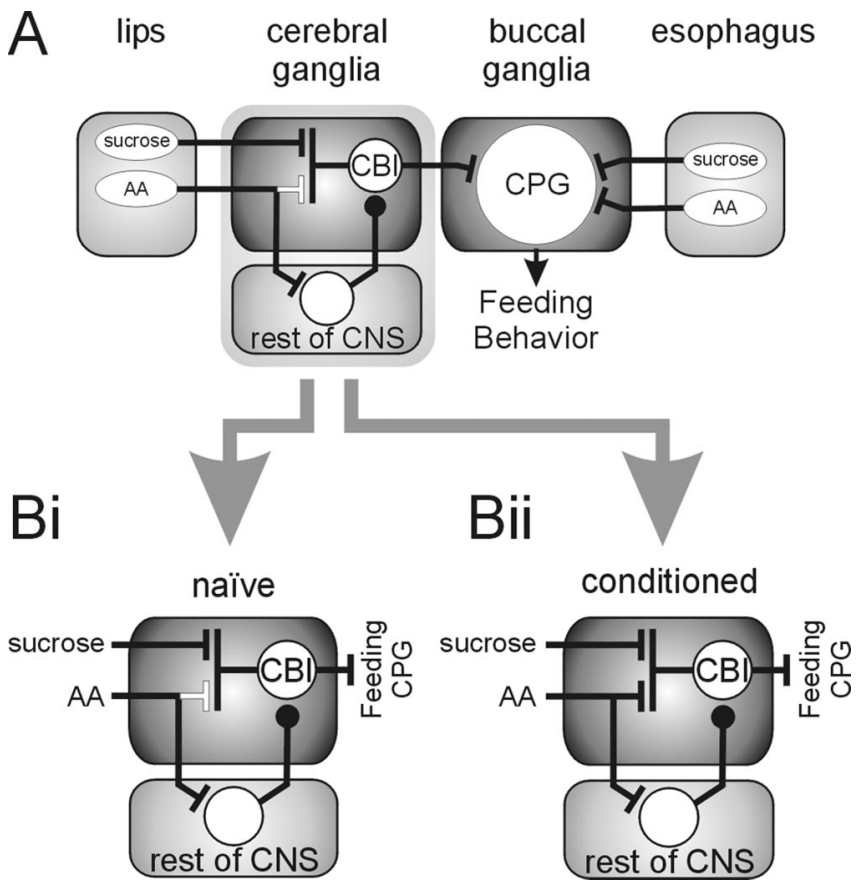

Figure 5. Network model for processing of chemosensory information in Lymnaea showing conditioning-induced changes. $\boldsymbol{A}$, Schematic overview of neuronal pathways involved in the processing of chemosensory information and learning. Sensory information from chemoreceptors located on the lips (external) and the esophagus (internal) are processed by anatomically distinct neuronal pathways. US sucrose and CS amyl acetate (AA) information from the lips is integrated in the cerebral ganglia. In contrast, US and CS information from the esophagus enters the buccal ganglia directly, and both stimuli activate the feeding central pattern generator $(C P G)$, increasing feeding behavior. Black lines, Functional connections; white lines, nonfunctional connections. Bars, Excitatory synapses; circles, inhibitory synapses. Bi, Details of chemosensory integration in cerebral ganglia of naive animals. US application leads to the activation of CBIs that are command neurons for feeding behavior resulting in the stimulation of the feeding central pattern generator. In contrast, the CS application results in the activation of neuronal elements in the rest of the CNS that suppress feeding central pattern generator activity by inhibiting $\mathrm{CBI}$ activity that provides the drive for spontaneous feeding activity. Bii, Conditioning-induced changes in the processing of CS information in the cerebral ganglia. Classical reward conditioning of Lymnaea feeding behavior leads to the formation of a new functional excitatory pathway between CS-sensitive chemoreceptors in the lips and CBIs, thus enabling CS application to activate CBIs and stimulate feeding activity. Note that the preexisting CS processing pathways in naive animals are unaltered by conditioning and contribute to the final behavioral effect of the $C S$ in conditioned animals.

more, a direct comparison of the effects of CS application to the lips shows that the net feeding stimulatory effect is significantly larger in conditioned than in control reduced preparations $(\Delta$ fictive feeding, conditioned, $2.1 \pm 0.4$ cycles $/ 2 \mathrm{~min}, n=20$; control, $0.5 \pm 0.5$ cycles $/ 2 \mathrm{~min}, n=15$; $t$ test, $p<0.05)$. This provides direct evidence that conditioning changes the response to CS application to the lips, so that it can activate a previously ineffective lip-cerebral-buccal pathway that now mediates a significant feeding stimulatory effect. It is also noteworthy that the size of the conditioned fictive feeding response after CS application to the lips $(2.1 \pm 0.4$ cycles $/ 2 \mathrm{~min} ; n=20)$ is similar to the changes in fictive feeding rate induced by US application to the lips in the same preparations from naive $(2.2 \pm 0.6$ cycles $/ 2 \mathrm{~min} ; n=13)$, control $(2.8 \pm 1.4 ; n=10)$, and conditioned $(1.4 \pm 0.6$ cycles $/ 2$ min; $n=14$; ANOVA, $F=0.5, p>0.5)$ animals. This suggests that the modified CS pathway accesses the same final common pathway for feeding activation (Fig. 5Bii) as the US pathways.

This CS feeding stimulatory pathway is also active in the whole CNS preparation of conditioned animals, in which its action counters the feeding inhibitory effect of the CS in unpaired control preparations, resulting in its reversal ( $\Delta$ fictive feeding, control animals, $-1.5 \pm 0.7$ cycles $/ 2 \mathrm{~min}, n=17$; conditioned animals, $0.6 \pm 0.3$ cycles $/ 2$ min, $n=15 ; t$ test, $p<0.001$ ) (Fig. $4 C$ ).

\section{Appetitive conditioning does not change the behavioral effect of the CS through existing pathways}

Additional analysis of the data from these experiments on reduced and whole brain preparations also provided evidence that the strength of the inhibitory component of the lip CS pathway is not changed by conditioning (Fig. $4 D$ ). The contribution of the inhibitory component $(\Delta i)$ to the overall effect of the CS on feeding activity was calculated as the absolute difference between the change in fictive feeding rate produced by CS application to the lips in whole and reduced CNS preparations (Fig. $4 \mathrm{D}$ left; for additional justification, see figure legend). On the assumption that this inhibitory component is not changed by conditioning, adding the $\Delta i$ value to the CS response in whole CNS preparations from conditioned animals should predict the conditioned response in the reduced CNS preparation (Fig. $4 D$, right). A comparison of the observed CS response in reduced CNS preparations from conditioned animals with the predicted conditioned CS response shows no significant differences ( $t$ test, $p>0.05$ ), supporting the hypothesis that conditioning is not affecting the existing inhibitory CS pathway. An alternative analysis using a two-way ANOVA of the effects of CS application to the lips on feeding activity in whole and reduced CNS preparations from control and conditioned animals also reveals no significant interaction effect between the type of preparation (factor 1, whole vs reduced CNS) and the type of animal (factor 2, control vs conditioned; $F=0.27, p>0.05$ ). If changes in the inhibitory CS pathway had contributed to the conditioned CS response, it would have resulted in a significant interaction effect, because the inhibitory CS pathway is only present in the whole CNS but not in the reduced CNS preparation.

Conditioning also did not change the feeding stimulatory response attributable to esophageal CS application. CS application to the esophagus alone in whole semi-intact preparations increased fictive feeding activity in both control and conditioned animals from $3.1 \pm 0.6$ cycles $/ 2 \min (n=8)$ and $3.8 \pm 0.6$ cycles/2 $\min (n=7)$, respectively, to $5.7 \pm 0.4$ and $7.1 \pm 0.9$ cycles $/ 2 \mathrm{~min}$ (paired $t$ tests, $p<0.05$ ). There was no difference in the effect on fictive feeding in the two groups ( $\Delta$ fictive feeding, control animals, $2.6 \pm 0.6$ cycles $/ 2 \mathrm{~min}$ vs conditioned animals, $3.3 \pm 1.0$ cycles $/ 2 \mathrm{~min} ; t$ test, $p>0.05$ ).

\section{Discussion}

Our results demonstrate the existence of two independent CS processing pathways with significant functional effects on feeding behavior in naive animals. The existence of these two pathways could suggest that appetitive conditioning is a form of alpha conditioning (Dudai, 1989), in which the conditioned response results from changes in existing CS processing pathways. Hypothetically, either a weakening of the feeding inhibitory CS effect on external chemosensory structures (lip) or a strengthening of the feeding stimulatory CS effect on internal (esophagus) chemosensory structures could account for the conditioned response. However, our studies revealed no significant changes in the effects of either of these two pathways on feeding behavior. Instead, we identified a third CS processing pathway that is modified in conditioned animals so that it mediates a significant feeding stimulatory effect in response to external CS application. Hints of this third pathway may have been present in reduced CNS prepara- 
tions from both naive and control animals, in which CS application to the lips slightly enhanced fictive feeding activity (Figs. $3 A$ ii, $4 B$ ). However, this potential unconditioned effect was at best very weak. Even combining the data from naive and control reduced CNS preparations failed to reveal a statistically significant enhancement of fictive feeding by the CS ( $\Delta$ fictive feeding, $0.4 \pm 0.3$ cycles $/ 2$ min; paired $t$ test, $p>0.05 ; n=49$ ). Although this data does not exclude the possibility of a feeding stimulatory lip-cerebral-buccal CS pathway in naive animals, our data show that this pathway is functionally inoperative in naive animals. Thus, we conclude that the enhanced CS feeding response after conditioning is attributable to modifications in this lip stimulatory pathway rather than changes in the lip inhibitory and esophageal excitatory pathways that provide the main contribution to CS responses in naive animals.

Although this study has been conducted at the systems level, we can propose a minimal network model to account for associative conditioning (Fig. 5). This model is built on our detailed knowledge of the feeding circuitry, which includes the cerebralbuccal interneurons (CBIs) (Kemenes et al., 2001; Straub et al., 2004). These are a group of command neurons located in the cerebral ganglia that project to the buccal ganglia, in which they can activate the feeding central pattern generator (Fig. $5 A$ ). We propose that, in conditioned animals, these neurons are recruited to the feeding stimulatory pathway and enhance their responsiveness to the CS attributable to modifications in the synaptic connections between CS-sensitive pathways and the CBIs (Fig. $5 B i, B i i)$. This model is consistent with data from our previous work recording axonal CBI activity in the connective between the cerebral and buccal ganglia in control and conditioned animals (Straub et al., 2004). This work showed that the CBIs responded with an increase in activity to lip application of the US and the CS (after conditioning) and identified the cerebral ganglia as a main site of synaptic plasticity underlying chemical conditioning. Furthermore, intracellular recordings from an identified CBI, the cerebral ventral 1 neuron (CV1), have shown that these neurons only respond to the CS after successful appetitive conditioning with no synaptic response before conditioning (Whelan and McCrohan, 1996; Kemenes et al., 2002). Similarly, in the honey bee, activity patterns of a multimodal integrating neuron, PE1, in response to a CS have been shown to be modulated by conditioning (Mauelshagen, 1993). The similarity of the strength of the fictive feeding response to the CS after conditioning and to the US in the present study also supports the hypothesis that the CBIs are the common final pathway for the activation of feeding.

The enhanced recruitment of CBIs to the CS response could be the result of changes in their intrinsic properties that increase their responsiveness to the CS. This has been shown to be an important contributor to tactile conditioning of Lymnaea feeding behavior, in which conditioning results in a persistent depolarization of the CV1 membrane potential (Jones et al., 2003). However, no such persistent depolarization of the CV1 membrane potential has been found after chemical conditioning in Lymnaea (Kemenes et al., 2000). Therefore, we conclude that the chemical conditioning-induced changes are more likely to involve changes in the functional connectivity of the CS processing pathways. We suggest that this could be the result of the formation of novel synaptic connections between the CS pathways and the CBIs. Alternatively, existing synapses may be nonfunctional or too weak to mediate a strong effect on feeding activity in naive animals before conditioning. Strengthening of weak synapses has been demonstrated to underlie various conditioning paradigms (Kandel and Schwartz, 1982; Glanzman, 1995; Hawkins et al.,
1998; Antonov et al., 2003; Roberts and Glanzman, 2003; Wickens et al., 2003; Crow, 2004), whereas the activation of silent synapses has been suggested to contribute to long-term potentiation (Montgomery et al., 2001; Voronin and Cherubini, 2004). Regardless of the underlying mechanism, our results suggest that conditioning leads to the creation of a functionally new pathway, probably attributable to the enhanced recruitment of CBIs to the CS processing pathway, which enables the CS to activate feeding after conditioning.

This model circuitry provides the mechanism for the integration of modified and unmodified CS processing pathways and accounts for the conditioned response in the intact animal. In naive snails, the stimulation of the lips by the high concentrations of the CS suppresses spontaneous feeding and consequently prevents the activation of the esophageal excitatory pathway, resulting in the overall reduction of feeding activity. At lower CS concentrations, however, lip stimulation does not suppress spontaneous feeding and the internalization of amyl acetate activates the low-threshold excitatory esophageal pathway, leading to the stimulation of feeding activity. In conditioned snails, enhanced activation of the lip excitatory pathway also enables high CS concentrations to have feeding stimulatory effects because activation of this pathway offsets the inhibitory effect of the CS via the lips. It thereby allows the esophageal pathway to dominate and initiate conditioned feeding. Thus, the conditioned behavior requires the parallel activation and integration of pathways that are both unmodified and modified by training.

\section{References}

Alexander Jr J, Audesirk TE, Audesirk GJ (1984) One-trial reward learning in the snail Lymnaea stagnalis. J Neurobiol 15:67-72.

Alexander Jr JE, Audesirk TE, Audesirk GJ (1982) Rapid, nonaversive conditioning in a freshwater gastropod. II. Effects of temporal relationships on learning. Behav Neural Biol 36:391-402.

Antonov I, Antonova I, Kandel ER, Hawkins RD (2001) The contribution of activity-dependent synaptic plasticity to classical conditioning in Aplysia. J Neurosci 21:6413-6422.

Antonov I, Antonova I, Kandel ER, Hawkins RD (2003) Activity-dependent presynaptic facilitation and hebbian LTP are both required and interact during classical conditioning in Aplysia. Neuron 37:135-147.

Audesirk TE, Alexander Jr JE, Audesirk GJ, Moyer CM (1982) Rapid, nonaversive conditioning in a freshwater gastropod. I. Effects of age and motivation. Behav Neural Biol 36:379-390.

Benjamin PR, Elliott CJH (1989) Snail feeding oscillator: the central pattern generator and its control by modulatory interneurons. In: Neuronal and cellular oscillators (Jacklet J, ed). New York: Dekker.

Benjamin PR, Staras K, Kemenes G (2000) A systems approach to the cellular analysis of associative learning in the pond snail Lymnaea. Learn Mem 7:124-131.

Crow T (2004) Pavlovian conditioning of Hermissenda: current cellular, molecular, and circuit perspectives. Learn Mem 11:229-238.

Dudai Y (1989) The neurobiology of memory: concepts, findings, trends. New York: Oxford UP.

Ferguson GP, Benjamin PR (1991) The whole-body withdrawal response of Lymnaea stagnalis. I. Identification of central motoneurones and muscles. J Exp Biol 158:63-95.

Fulton D, Kemenes I, Andrew RJ, Benjamin PR (2005) A single timewindow for protein synthesis-dependent long-term memory formation after one-trial appetitive conditioning. Eur J Neurosci 21:1347-1358.

Glanzman DL (1995) The cellular basis of classical conditioning in Aplysia californica - it's less simple than you think. Trends Neurosci 18:30-36.

Hawkins RD, Greene W, Kandel ER (1998) Classical conditioning, differential conditioning, and second-order conditioning of the Aplysia gillwithdrawal reflex in a simplified mantle organ preparation. Behav Neurosci 112:636-645.

Inoue T, Takasaki M, Lukowiak K, Syed NI (1996) Inhibition of the respiratory pattern-generating neurons by an identified whole-body withdrawal interneuron of Lymnaea stagnalis. J Exp Biol 199:1887-1898. 
Jones NG, Kemenes I, Kemenes G, Benjamin PR (2003) A persistent cellular change in a single modulatory neuron contributes to associative longterm memory. Curr Biol 13:1064-1069.

Kandel ER, Schwartz JH (1982) Molecular biology of learning: modulation of transmitter release. Science 218:433-443.

Kemenes G, Elliott CJH, Benjamin PR (1986) Chemical and tactile inputs to Lymnaea feeding system-effects on behavior and neural circuitry. J Exp Biol 122:113-137.

Kemenes G, Jones NG, Staras K, Kemenes I, O’Shea M, Benjamin PR (2000) Cellular changes in the feeding system after tactile and chemical appetitive classical conditioning in Lymnaea. Eur J Neurosci 12:93.

Kemenes G, Staras K, Benjamin PR (2001) Multiple types of control by identified interneurons in a sensory-activated rhythmic motor pattern. J Neurosci 21:2903-2911.

Kemenes I, Kemenes G, Andrew RJ, Benjamin PR, O’Shea M (2002) Critical time-window for NO-cGMP-dependent long-term memory formation after one-trial appetitive conditioning. J Neurosci 22:1414-1425.

Kyriakides MA, McCrohan CR (1988) Central coordination of buccal and pedal neuronal activity in the pond snail Lymnaea stagnalis. J Exp Biol 136:103-123.

Mauelshagen J (1993) Neural correlates of olfactory learning paradigms in an identified neuron in the honeybee brain. J Neurophysiol 69:609-625.

Montgomery JM, Pavlidis P, Madison DV (2001) Pair recordings reveal allsilent synaptic connections and the postsynaptic expression of long-term potentiation. Neuron 29:691-701.

Ribeiro MJ, Serfozo Z, Papp A, Kemenes I, O’Shea M, Yin JCP, Benjamin PR, Kemenes G (2003) Cyclic AMP response element-binding (CREB)-like proteins in a molluscan brain: cellular localization and learning-induced phosphorylation. Eur J Neurosci 18:1223-1234.

Roberts AC, Glanzman DL (2003) Learning in Aplysia: looking at synaptic plasticity from both sides. Trends Neurosci 26:662-670.

Rose RM, Benjamin PR (1979) The relationship of the central motor pattern to the feeding cycle of Lymnaea stagnalis. J Exp Biol 80:137-163.

Straub VA, Staras K, Kemenes G, Benjamin PR (2002) Endogenous and network properties of Lymnaea feeding central pattern generator interneurons. J Neurophysiol 88:1569-1583.

Straub VA, Kemenes I, Styles BJ, O’Shea M, Benjamin PR (2003) Contribution of external and internal chemosensory information to the activation of feeding and appetitive conditioning. Soc Neurosci Abstr 29:520.19.

Straub VA, Styles BJ, Ireland JS, O'Shea M, Benjamin PR (2004) Central localization of plasticity involved in appetitive conditioning in Lymnaea. Learn Mem 11:787-793.

Thompson RF (2005) In search of memory traces. Annu Rev Psychol 56:1-23.

Voronin LL, Cherubini E (2004) "Deaf, mute and whispering" silent synapses: their role in synaptic plasticity. J Physiol (Lond) 557:3-12.

Whelan HA, McCrohan CR (1996) Food-related conditioning and neuronal correlates in the freshwater snail Lymnaea stagnalis. J Molluscan Stud 62:483-494.

Wickens JR, Reynolds JN, Hyland BI (2003) Neural mechanisms of rewardrelated motor learning. Curr Opin Neurobiol 13:685-690.

Winlow W, Moroz LL, Syed NI (1992) Mechanisms of behavioural selection in Lymnaea stagnalis. In: Neurobiology of motor programme selection (Kien J, McCrohan CR, Winlow W, eds), pp 52-72. New York: Pergammon. 